\title{
GENERALIZED ARCHIMEDEAN GROUPS
}

\author{
BY \\ ELIAS ZAKON
}

Introduction. In a previous paper [3], the concept of archimedean group was generalized so as to obtain a larger class of groups that were referred to as regularly ordered. As it was shown in [3], these groups, unlike the archimedean ones, admit a formalization in the lower predicate calculus (LPC) of formal logic; yet they cannot be distinguished from archimedean groups by any properties formalizable in the LPC. Thus they can serve as an LPC-substitute for archimedean groups. This result was, however, based on several theorems stated without proof. It is our aim now to supply the proofs of these theorems. Another objective of this paper consists in giving a unified algebraic approach to regularly ordered groups, as a counterpart to the metamathematical study presented in [3].

Throughout this paper, the term "ordered group" stands for "totally ordered (and, hence, torsion-free) additive abelian group other than $\{0\} . "$ Such a group, $A$, is said to be discretely or densely ordered according as it does, or does not, contain a smallest positive element (called its unit, i). A subset $S \subseteq A$ is called an interval if the relations $a<x<b, a, b \in S, x \in A$ always imply $x \in S$. An interval is referred to as infinite if the number of its elements is infinite. Whereas in [3] the concept of a regularly ordered group was defined separately for discretely and densely ordered groups, we now give a unified definition; its equivalence with that given in [3] will be proved later.

0.1 . Definition. An ordered group $A$ is said to be regularly ordered, with respect to an integer $n>0$ (briefly, " $n$-regular"), if every infinite interval of $A$ contains at least one (and, hence, infinitely many) elements divisible by $n .\left({ }^{1}\right)$ If this holds for every $n$, we simply say that $A$ is regularly ordered. $A$ is referred to as regularly discrete (regularly dense) if its ordering is both regular and discrete (regular and dense, respectively).

From this definition we immediately derive the following corollary, to be used later.

0.2 . Corollary. Let a be an element of a regularly ordered group $A$, and let $k, r$ be positive integers. Then every infinite interval $S$ of $A$ contains an element $x$ of the form $x=k y$, with $y \equiv a(\bmod r), y \in A$.

Received by the editors March 2, 1960 and, in revised form, September 27, 1960.

(1) As usual, we say that an element $x$ is divisible by $n$ (briefly, " $n$-divisible") in a group $A$, if $A$ contains an element $z$ with $x=n z$. Two elements $x, y \in A$ are said to be congruent modulo $n$ (briefly, " $n$-congruent") in $A$, if $x-y$ is divisible by $n$. We then write $x \equiv y(\bmod n)$ in $A$. The negation of this formula is $x \neq y(\bmod n)$ in $A$ ( ${ }^{\prime} x$ and $y$ are $n$-incongruent in $\left.A^{n}\right)$. 
Proof. By $k$-regularity, $S$ contains infinitely many elements $x$ which are divisible by $k$. If $x$ is treated as a variable ranging over all such elements of $S$, then, as is easily checked, the set of all elements of the form $x / k-a$ is, likewise, an infinite interval of $A$. Hence, by $r$-regularity, it contains an $r$-divisible element, so that we have $x / k \equiv a(\bmod r)$ for some $x \in S$. It now suffices to set $x / k=y$, to obtain the required result.

Some further preliminary definitions and corollaries are given in $\$ 1$.

1. Preliminaries.

I. As in [3], we define the $n$th congruence invariant of an abelian group $A$, denoted by $[n] A$ or, briefly, by $[n]$, to be the maximum (possibly infinite) number of $n$-incongruent elements in $A .\left(^{2}\right)$ If $n$ is a prime, $[n]$ is called a prime invariant. In the infinite case, we set $[n]=\infty$, without distinguishing between infinities of different cardinalities $\left({ }^{3}\right)$.

1.1. CoRollary. If a group $A$ is isomorphic with the additive group of all integers, then $[n] A=n, n=1,2,3, \cdots$.

In fact, for every $n$, the group $A$ splits into exactly $n$ residue classes modulo $n$; i.e., the quotient group $A / n A$ has order $n$ (cf. footnote 2).

II. Let $A$ be an additive abelian group, $S$ its subgroup, and $n>0$ an integer. We shall say that $S$ is $n$-serving in $A$ if, for all $x, y \in S$, the relation $x \equiv y(\bmod n)$ holds in $S$ whenever it holds in $A$. If this is true for every $n$, we simply say that $S$ is serving in $A$ (Prüfer, [1]). $S$ is said to be $n$-basic in $A$, if every element of $A$ is $n$-congruent to some element of $S$. Again, if this holds for every $n$, we say that $S$ is basic in $A$.

1.2. Corollary. Let $A_{0}$ and $A\left(A_{0} \subseteq A\right)$ be regularly discrete groups with one and the same unit $\mathrm{i}$. Then $A_{0}$ is basic and serving in $A$.

Proof. Let $A_{1}$ be the subgroup generated by the unit $\mathrm{i}$, and let $a \in A$. Clearly, all elements of the form $a-k \cdot 1(k=1,2,3, \cdots)$ constitute an infinite interval of $A$ which, by $n$-regularity ( $n$ being any positive integer), must contain an $n$-divisible element, say $a-k_{0} \cdot \dot{1}$, so that $a \equiv k_{0} \cdot \dot{1}(\bmod n)$, and $k_{0} \cdot \mathrm{i} \in A_{1}$. Thus every element of $A$ is $n$-congruent to some element of $A_{1}$. Since $n$ is arbitrary here, $A_{1}$ is basic in $A$ and, similarly, $A_{0}$ is basic in $A$. Moreover, if $a \in A_{0}$, the congruence $a \equiv k_{0} \cdot \mathrm{i}(\bmod n)$ easily implies that $n$ divides $a$ (in both $A_{0}$ and $A$ ) if and only if $n$ divides the integer $k_{0}$. It follows that $n$ divides $a$ either in both $A_{0}$ and $A$, or in none of these groups. This shows that $A_{0}$ is serving in $A$, as asserted.

\subsection{Corollary. A subgroup $S$ of a torsion-free abelian group $A$ is serving}

(2) This maximum number always exists and is unique. In fact, as is readily seen, $[n] A$ coincides with the (finite or infinite) order of the quotient group $A / n A$, where $n A$ is the subgroup of all elements divisible by $n$.

(3) The symbol $\infty$ will be treated as a positive number with the usual conventions as to operations and inequalities. In particular, we set $\log _{p} \infty=\infty$ for every $p>1$. 
(basic) in $A$ if and only if, for every prime $p, S$ is p-serving (p-basic) in $A$. (For basic subgroups, this holds also if $A$ is not torsion-free.)

Proof. Finite induction easily shows that, whenever $S$ is $p$-serving, it is also $p^{k}$-serving $(k=1,2,3, \cdots)$. It is also readily seen that, whenever $S$ is both $m$-serving and $n$-serving ( $m$ and $n$ being relatively prime), then $S$ is also $(m n)$-serving. Similarly for basic subgroups. The required result then is obtained by considering each integer $n$ as factored into prime powers.

1.4. Corollary. Let $S$ be a subgroup of an abelian group $A$, and $n>0$ an integer. Then, if $S$ is $n$-serving in $A$, we have $[n] S \leqq[n] A$. If, however, $S$ is $n$-basic in $A$, we have $[n] S \geqq[n] A$.

For, let $[n] S=m \leqq \infty$; i.e., $S$ contains exactly $m$ elements incongruent modulo $n$. If $S$ is $n$-serving, these elements are $n$-incongruent in $A$ as well, whence $[n] \geqq m=[n] S$. If, however, $S$ is $n$-basic, then, clearly, $A$ cannot contain more $n$-incongruent elements than $S$; hence $[n] A \leqq[n] S$.

1.5. Corollary. If $A$ is discretely ordered, then $[n] A \geqq n, n=1,2,3, \cdots$.

In fact, let $A_{1}$ be the subgroup generated by the unit $\mathrm{i}$ of $A$. Then, as is readily seen, $A_{1}$ is serving in $A$ and isomorphic with the ordered group of all integers. By 1.1 and 1.4 , we then have $n=[n] A_{1} \leqq[n] A$, as asserted.

III. A subset $S$ of an abelian group $A$ is said to be $n$-independent (in $A$ ) if, for any finite number $m$ of distinct elements $x_{1}, x_{2}, \cdots, x_{m} \in S$, and any integers $q_{1}, q_{2}, \cdots, q_{m}$, the relation $\sum_{i=1}^{m} q_{i} x_{i} \equiv 0(\bmod n)$ in $A$ always implies $q_{i} \equiv 0(\bmod n), i=1,2, \cdots, m . S$ is referred to as maximal if it not contained in any other $n$-independent subset of $A$. The existence of (possibly empty) maximal $n$-independent subsets, for every $n$, is easily proved by Zorn's lemma. Also easily derived is the following

1.6. COROLLARY. In a torsion-free abelian group, every $n$-independent subset is also $n^{k}$-independent $(k=1,2,3, \cdots)$.

1.7. Corollary. If an abelian group $A$ is not divisible ${ }^{(4)}$ by a prime $p$, then $A$ contains at least one nonempty maximal p-independent subset.

Proof. Take any element $x \in A$ not divisible by $p$. Then $\{x\}$ is clearly a nonempty $p$-independent subset of $A$. The rest follows by Zorn's lemma.

1.8. Corollary. Let $A$ be an abelian group, and $p$ a prime. Then all maximal p-independent subsets of $A$ contain exactly the same (finite or infinite) number of elements. This number (called the pth prime rank of $A$ ) equals $\log _{p}[p] A$.

Proof. Let $S$ be a maximal $p$-independent subset of $A$, and let $m$ be the

(4) We say that a group $A$ is divisible by an integer $n>0$, or that $n$ divides $A$, if all elements of $A$ are $n$-divisible (in $A$ ). If this holds for every $n, A$ is referred to as (completely) divisible. 
number of its elements. Then it easily follows that $A$ contains exactly $p^{m}$ $p$-incongruent elements $\left(^{5}\right)$. In our notation, this means that $[p] A=p^{m}$, or $m=\log _{p}[p] A$. As the value of $[p] A$ is uniquely determined, all is proved.

IV. Given a subgroup $A_{0}$ of an abelian group $A$, and $c_{0} \in A$, we define the $c_{0}$-extension of $A_{0}$ in $A$ to be the subgroup of all elements $x \in A$ satisfying equations of the form $t x=s c_{0}+b$, with $b \in A_{0}$ and $t, s$ integers $(t>0)$.

1.9. Corollary. Let $A_{1}$ be the $c_{0}$-extension of a subgroup $A_{0}$ in $A$. Then:

(i) $A_{1}$ is a serving subgroup of $A$ which contains $A_{0}$ and $c_{0}$.

(ii) $A_{0}$ is serving in $A_{1}$ if and only if $A_{0}$ is serving in $A$.

(iii) If $A_{0}$ and $A$ are regularly discrete, and have the same unit $\mathrm{i}$, then $A_{1}$ has the same unit and, moreover, $[n] A_{0}=[n] A_{1}=[n] A, n=1,2,3, \cdots$.

Proof. Assertions (i) and (ii) easily follow from the definition (IV). Since $A_{0} \subseteq A_{1}$, we see, in case (iii), that $\mathrm{i} \in A_{1}$, so that $A_{1}$ has the same unit. By $1.2, A_{0}$ is basic and serving in $A$. From this, and (i), (ii), it follows, by 1.4 , that $[n] A_{0}=[n] A_{1}=[n] A$, for all $n$, as required.

$\mathrm{V}$. As is well known, every torsion-free (ordered) group $A$ can be embedded in a smallest divisible extension, i.e., a smallest divisible group which contains it and is, likewise, torsion-free (ordered, respectively). The elements of that extension (which is unique, up to isomorphism) are all of the form $x / n$, with $x \in A$ and $n$ a positive integer $\left({ }^{6}\right)$.

VI. For further references, we list some familiar properties of direct sums.

1.10. Theorem. Let $M$ be the direct sum of two abelian groups, $A$ and $B$, with $A \cap B=\{0\}$. Then: (a) If $A$ and $B$ are torsion-free (ordered), so is $M$.

(b) Every element $u \in M$ admits a unique representation $u=x+y$ $(x \in A, y \in B$ ).

(c) For any $x \in A$ and $y \in B$, we have $x+y \equiv 0(\bmod n)$ if and only if $x \equiv 0(\bmod n)$ in $A$, and $y \equiv 0(\bmod n)$ in $B$.

(d) $A$ and $B$ are serving subgroups of $M$.

Note. If $A$ and $B$ are ordered, $M$ is assumed to be ordered lexicographically.

2. The class of regularly ordered groups. We shall now study more closely the class of regularly ordered groups.

2.1. Theorem. (a) $A$ densely ordered group $A$ is regularly dense if and only if, for every integer $n>0$, and any $a, b \in A(a<b)$, there is an $x \in A$ with $a<n x<b$.

(b) $A$ discretely ordered group $A$ is regularly discrete if and only if $[n] A=n$, for every integer $n>0$.

(๖) For the method of the proof of this assertion, see e.g., [4, p. 214].

( $)$ All these assertions can be proved by a procedure analogous to the familiar process of forming the quotient field of an integral domain. 
Proof. Assertion (a) follows immediately from Definition 0.1 if one considers that, in a densely ordered group, every interval with endpoints $a, b$ $(a<b)$ is infinite. Thus we proceed to prove (b).

I. Necessity. Let $A$ be regularly discrete; further, let $A_{1}$ be the subgroup generated by the unit $\mathrm{i}$ of $A$. Since $A_{1}$ is isomorphic to the group of all integers, we have $[n] A_{1}=n, n=1,2,3, \cdots$ (see 1.1). Moreover, by $1.2, A_{1}$ is basic in $A$. Hence, by 1.4 , and $1.5, n=[n] A_{1} \geqq[n] A \geqq n$, for all $n$, as required.

II. Sufficiency. Suppose that $[n] A=n, n=1,2, \cdots$, further, let $S$ be any infinite interval of $A$, and $a \in S$. As is readily seen, $S$ contains either all elements of the form $a+k \cdot \mathbf{i}$, or all elements of the form $a-k \cdot \mathbf{i}(k=1,2,3, \cdots)$, say the latter. Now consider the $n$ elements $k \cdot 1(k=1,2,3, \cdots, n)$. They are clearly $n$-incongruent with each other. On the other hand, the assumption $[n] A=n$ implies that $A$ cannot contain more than $n$ elements incongruent modulo $n$. Hence the element $a$ (selected above) must be $n$-congruent with one of the $n$ elements $k \cdot \mathrm{i}(k=1,2, \cdots, n)$. Thus we have $a \equiv k_{0} \cdot \mathrm{i}(\bmod n)$ for some $k_{0}>0$. But then the element $a-k_{0} \cdot \mathrm{i} \in S$ is divisible by $n$. Thus every infinite interval $S$ of $A$ contains an $n$-divisible element, and all is proved.

2.1.1. Corollary. Let $A_{0}$ and $A\left(A_{0} \subseteq A\right)$ be regularly discrete groups having the same unit $\mathrm{i}$, and let $c_{0} \in A$. Then the $c_{0}$-extension of $A_{0}$ in $A$ is, likewise, regularly discrete and has the same unit $\mathrm{i}$.

For the proof, combine 2.1(b) with 1.9(iii).

2.2. THEOREM. For an ordered group $A$ to be regularly ordered, it suffices that $A$ be p-regular for every prime $p$.

For the proof, we first establish the following fact.

2.2.1. If $A$ is both m-regular and n-regular, then $A$ is (mn)-regular.

Let $S$ be an infinite interval of $A$. Due to $m$-regularity, $S$ contains infinitely many elements divisible by $m$. If $x$ is a variable ranging over all $m$ divisible elements of $S$, then, as is easily seen, all quotients $x / m$ again constitute an infinite interval of $A$. One of these quotients, say $x_{0} / m\left(x_{0} \in S\right)$, must be divisible by $n$, due to the $n$-regularity of $A$. Hence the element $x_{0} \in S$ is divisible by $m n$. This establishes 2.2.1. To complete the proof of 2.2 , one only has to consider each integer as factored into prime powers and apply 2.2.1 to the arising products.

Theorems 2.1 and 2.2, when combined, show that our Definition 0.1 is equivalent to the definition of regularly ordered groups given in $[3,(3.3)]$.

We now proceed to prove some theorems announced in [3].

2.3. Lemma. Let $b>0$ be an element of a densely ordered group $A$. Then, for every integer $n>0$, there is an element $x \in A$ such that $0<n x<b$.

For the proof, select any $n+1$ elements $x_{i} \in A$ such that $0<x_{1}<x_{2}<\ldots$ $<x_{n+1}<b$, and set $x=\min \left(x_{i+1}-x_{i}\right)$. Then $x$ is the required element. 


\subsection{TheOREM. All archimedean groups are regularly ordered.}

Proof. Let $A$ be an archimedean group. If $A$ is discretely ordered, then, as is readily seen, the archimedean property implies that all elements of $A$ are integral multiples of its unit $i$. Hence $A$ is isomorphic with the additive group of all integers, and our assertion follows from 1.1 and 2.1(b). Now suppose that $A$ is densely ordered, and let $S$ be an infinite interval of $A$, and $n$ a positive integer. We shall show that $S$ contains an $n$-divisible element and thus complete the proof. Obviously, it suffices to consider the case $0 \notin S$. In this case, all elements of $S$ have the same sign, say positive. Let then $a, b \in S$, $0<a<b$. Taking in 2.3 for $b$ the minimum of $a$ and $b-a$, we see that there is an $x \in A$ with 2.4.1. $0<n x<a, 0<n x<b-a$.

The archimedean property entails that there are positive integers $m$ with $m n x>a$. Let $m_{0}$ be the least of them. Then it is easily deduced from 2.4.1 that $a<m_{0} n x<b$, so that, by the definition of an interval, the element $y=m_{0} n x$ belongs to $S$ (for so do $a$ and $b$ ). As $y$ is also divisible by $n$, all is proved.

From this theorem it follows, in particular, that every group consisting of real numbers in their natural order is regularly ordered (for it is archimedean). On the other hand, it is easy to give examples of regularly ordered groups which are not archimedean. Such is, for instance, the additive group of all complex numbers, ordered lexicographically, and, more generally, every nonarchimedean divisible group (ordered divisible groups are, obviously, regularly dense). The direct sum of any ordered divisible groups is regularly dense, though not archimedean, under lexicographic ordering. There are also regularly discrete nonarchimedean groups. Such is, for instance, the lexicographically ordered additive group of all complex numbers $a+b i$ with $b$ an integer. Thus the class of all regularly ordered groups is more general than that of all archimedean groups. The following existence theorem is important.

2.5. Theorem. Let $p_{1}, p_{2}, \cdots, p_{n}, \cdots$ be the ascending sequence of all primes; further, let $m_{1}, m_{2}, \cdots, m_{n}, \cdots$ be an arbitrary sequence in which each $m_{n}$ is either a non-negative integer or $\infty$. Then there always exists a densely ordered archimedean (and, hence, regularly dense) group $M$ such that

$$
\left[p_{n}\right] M=p_{n}^{m_{n}}, \quad n=1,2,3, \cdots
$$

Proof. As a preparatory step, we fix some transcendental real number $e$ and set up an infinite two-entry matrix $\left(a_{n j}\right), n, j=1,2,3, \cdots$, whose elements are either 0 or distinct powers of $e$, defined as follows:

(1) $a_{n j}=0$ whenever $j>m_{n}$, where $m_{n}$ is the $n$th term of the sequence $\left\{m_{n}\right\}$ specified in the theorem. If $m_{n}=0$, all $a_{n j}$ are 0 (for that $n$ ).

(2) If $j \leqq m_{n}$, we set $a_{n j}=e^{k_{n i}}$ where $k_{n j}$ is some non-negative integer. The 
exponents $k_{n j}$ are supposed to be distinct, but, otherwise, may be chosen arbitrarily. We shall consider the $k_{n j}$ and $a_{n j}$ as fixed.

Now let $R$ be the ordered additive group of all reals, and let $M$ be its subgroup generated by the set of all fractions of the form $a_{n j} / r$ where $a_{n j}$ is as defined above and $r$ is an integer not divisible by the prime $p_{n}$. As is easily seen, $M$ is a densely ordered archimedean group containing all $a_{n j}$. Moreover, using the fact that $e$ is transcendental, it is easy to show that the set $S_{n_{0}}$ of all nonzero elements $a_{n_{0} j}\left(j \leqq m_{n_{0}}\right)$ (i.e., the set of all nonzero elements in the $n_{0}$ th row of the matrix $\left.\left(a_{n j}\right)\right)$ is a maximal $p_{n_{0}}$-independent subset of $M$. It follows, by 1.8 , that $S_{n_{0}}$ contains exactly $\log _{p_{n_{0}}}\left[p_{n_{0}}\right] M$ elements. On the other hand, by the definition of the matrix $\left(a_{n j}\right)$, its $n_{0}$ th row contains exactly $m_{n_{0}}$ nonzero elements, and this is the number of elements in $S_{n_{0}}$. We conclude that $\log _{p_{n}}\left[p_{n}\right] M=m_{n}$, for every $n$; that is $\left[p_{n}\right] M=p_{n}^{m_{n}}$. Thus the group $M$ has all the required properties, and the theorem is proved. More concisely, it could be formulated thus:

$2.5^{\prime}$. For any choice of the prime ranks (see 1.8), there exists a densely ordered archimedean group $M$ with prime ranks so chosen.

Finally, Corollary 1.8 yields the following immediate inference.

2.6. THEOREM. In any abelian group, every prime invariant $\left[p_{n}\right]$ is a (possibly infinite) power of the corresponding prime $p_{n}$.

Theorems 2.4, 2.5 and 2.6 establish three of the results announced in [3] (where they appear as Theorems 3.4, 3.5 and 3.6, respectively). To prove the next of the required theorems (3.7 of [3]), we need some additional preliminary remarks concerning what we shall define as linear systems and closed subgroups. These remarks constitute a natural generalization of some concepts and propositions of linear algebra.

3. Linear systems. Closed subgroups.

I. As in [3], we define a linear system to be any finite system of linear equations, inequalities, congruences and (or) incongruences of the form

$$
\sum_{j=1}^{n} q_{i j} x_{j}=a_{i}, \quad i=1,2, \cdots, m_{1},
$$

$$
\sum_{j=1}^{n} q_{i j} x_{j}>a_{i}
$$

$$
i=m_{1}+1, m_{1}+2, \cdots, m_{2}
$$

$$
\sum_{j=1}^{n} q_{i j} x_{j} \equiv a_{i}\left(\bmod r_{i}\right)
$$

$$
i=m_{2}+1, m_{2}+2, \cdots, m_{3}
$$

$$
\sum_{i=1}^{n} q_{i j} x_{j} \not \equiv a_{i}\left(\bmod r_{i}\right)
$$$$
i=m_{3}+1, m_{3}+2, \cdots, m_{4},
$$

where the $q_{i j}$ and $r_{i}$ are integers $\left(r_{i}>0\right)$, the $x_{j}$ are unknowns or variables, and the $a_{i}$ are given elements of an ordered group $A$; the $a_{i}$ will be called 
the constants of the system. We shall denote by $\left(q_{i j}\right)$ the matrix of the coefficients $q_{i j}$ occurring in the equations 3.1 (if any), and by $\left(q_{i j}, a_{i}\right)$ the "extended" matrix which arises from $\left(q_{i j}\right)$ by adjoining the column of the constants $a_{i}$, $i=1,2, \cdots, m_{1}$. (Note that only the coefficients and constants of the equations 3.1, not those occurring in 3.2, 3.3 and 3.4, are spoken of.) A linear system with a nonempty set of equations 3.1 is said to be of type I, II or III, according as (I) the matrices $\left(q_{i j}\right)$ and $\left(q_{i j}, a_{i}\right)$ are of the same rank $n$ (=the number of the unknowns $x_{j}$ ), (II) the ranks of these matrices are equal, but less than $n$, or (III) their ranks are different. Two linear systems $L_{1}$ and $L_{2}$ will be referred to as conjugate if, whenever an ordered group $A$ contains their constants, either both $L_{1}$ and $L_{2}$, or none of them, can be solved in $A$. Equivalent systems are a special case.

II. Since every completely divisible torsion-free group is a vector space over the field of all rational numbers, it is clear that the ordinary theory of linear equations (in particular, Cramer's rule) is valid in every such group. Some familiar rules still remain valid for nondivisible groups and for more general linear systems, of the form 3.1-3.4. We list them here.

3.5. Theorem. (i) A linear system of type I has at most one solution in every ordered group $A$ containing its constants $a_{i}$. Moreover, the set of equations 3.1 contained in such a system is equivalent to some system of the form

$$
k x_{j}=c_{j},
$$$$
j=1,2, \cdots, n,
$$

where $k$ is a positive integer, and the $c_{j}$ are elements of the subgroup of $A$ generated by the constants $a_{i}$ occurring in 3.1 .

(ii) In every linear system of type II, with $a_{i} \in A$, the set of the equations 3.1 is equivalent to some system of the form

$$
k x_{j+m}=c_{j}+\sum_{i=1}^{m} k_{i j} x_{i}, \quad j=1,2, \cdots, n-m,
$$

where again $k$ is an integer $>0$, the $c_{j}$ are as above, and $m<n .\left(^{7}\right)$

(iii) No linear system of type III has a solution in any ordered group.

For the proof, it suffices to embed the group $A$ in its divisible extension $M$ and then apply Cramer's rule. The required results then easily follow if one takes into account that every element of $M$ has the form $a / q$ with $a \in A$ and $q$ an integer.

\subsection{Theorem. For every linear system $L$ of type II, there exists a conjugate}

$\left(^{7}\right)$ The integer $k$ in 3.5.1 and 3.5.2 is some multiple of the highest nonzero determinant of the matrix $\left(q_{i j}\right)$. To simplify notation, we have assumed that this determinant is in the right lower corner of the matrix. The elements $c_{j}$ are corresponding determinants (or their multiples) taken from $\left(q_{i j}, a_{j}\right)$, in accordance with Cramer's rule. Clearly, these determinants, when expanded, are "linear combinations" of the $a_{i}$. Hence the $c_{j}$ are in the subgroup generated by the $a_{i}$ (we shall briefly say that they are "generated by the $a_{i}{ }^{\prime}$ ). 
system $L^{\prime}$ with no equations 3.1 in it, and with all its constants in the subgroup generated by the constants $a_{i}$ of $L$.

Proof. The required system $L^{\prime}$ is obtained from $L$ in three steps:

(1) Using Theorem 3.5(ii), we replace the equations 3.1 of $L$ by 3.5.2, leaving the inequalities, congruences and incongruences (if any) unchanged. This, obviously, yields an equivalent and, hence, conjugate system (call it $\left.L_{1}\right)$.

(2) We now adjoin to $L_{1}$ the following system of congruences:

3.6.1.

$$
c_{j}+\sum_{i=1}^{m} k_{i j} x_{i} \equiv 0(\bmod k), \quad j=1,2, \cdots, n-m,
$$

where the $c_{j}, k_{i j}, k$ and the unknowns $x_{i}$ are the same as in 3.5.2. This again yields an equivalent system (call it $L_{2}$ ). For, every solution of $L_{2}$ clearly satisfies also $L_{1}$; conversely, every solution of $L_{1}$ satisfies, in particular, the equations 3.5.2 which imply 3.6.1, so that $L_{2}$ is solved as well. Moreover, as follows from Theorem 3.5, the constants of $L_{1}$ and $L_{2}$ are generated by those of $L$, as required.

(3) The last step is to remove the equations from $L_{2}$. We achieve this by using the equations 3.5.2, first of all, to eliminate the $n-m$ unknowns $x_{j+m}(j=1,2, \cdots, n-m)$ from the whole system $\left({ }^{8}\right)$. Having done so, we drop the equations 3.5.2, retaining, however, the congruences 3.6.1 instead. This leads to a new linear system $L_{3}$ with no equations in it and with its constants again generated by the constants of the original system $L$ (obviously, the elimination process described in footnote 8 satisfies these requirements). Moreover, every solution of $L_{2}$ clearly yields also a solution of $L_{3}$ and vice versa (for the whole process is reversible, due to the fact that ordered groups are torsion-free) $\left({ }^{9}\right)$. Thus $L_{2}$ and $L_{3}$ are conjugate, and $L_{3}$ is the required system $L^{\prime}$.

III. The remaining theorems of this and the next sections deal with what we shall now define as closed subgroups.

3.7. Definition. A subgroup $A_{0}$ of an ordered group $A$ is said to be closed in $A$ if every linear system, with its constants $a_{i}$ in $A_{0}$, can already be solved in $A_{0}$ whenever it has a solution in $A$. If this is true of linear systems of some specific kind, we shall say that $A_{0}$ is closed with respect to systems of that particular kind $\left({ }^{10}\right)$.

(8) This elimination process can be carried out also in nondivisible ordered (and, hence, torsion-free) groups by, say, multiplying all inequalities, congruences and incongruences of $L_{2}$ by $k$ and then replacing everywhere $k x_{j+m}$ by $c_{j}+\sum_{i-1}^{m} k_{i j} x_{i}$ (note that in the congruences and incongruences, the moduli $r_{i}$, too, must be multiplied by $k$ ).

( $\left.{ }^{9}\right)$ Note that the adjoined congruences 3.6.1 ensure, in their turn, the solvability of the equations 3.5.2. Thus any solution of $L_{3}$ yields a solution of $L_{2}$.

(10) Clearly, this is a generalization of Prüfer's concept of a serving subgroup which can now be defined as a subgroup closed with respect to all (single) equations of the form $q x=a$, or all congruences of the form $q x \equiv a(\bmod n)$. 
3.8. Theorem. Every serving subgroup $A_{0}$ of an ordered group $A$ is closed in $A$, with respect to all linear systems of type I.

Proof. Let $L$ be a linear system of type I, with its constants $a_{i}$ in $A_{0}$, and let $\left(b_{1}, b_{2}, \cdots, b_{n}\right)$ be its solution in $A$ (here, as before, $n$ is the number of the unknowns $x_{j}$ ). To prove the theorem, it suffices to show that all $b_{j}$ necessarily are elements of $A_{0}$. By 3.5(i), the $b_{j}$ must satisfy some equations of the form 3.5.1, where the $c_{j}$ are generated by the $a_{i}$. As all $a_{i}$ are in $A_{0}$, so are all $c_{j}$. Thus we have $k b_{j}=c_{j}, c_{j} \in A_{0}, j=1,2, \cdots, n$. It follows that the elements $c_{j}$ are divisible, in $A$, by the integer $k$. But, as $A_{0}$ is serving in $A$, this divisibility holds in $A_{0}$ as well. This means that the quotients $b_{j}=c_{j} / k$ are all in $A_{0}$, as required.

3.9. Theorem. For a subgroup $A_{0}$ of an ordered group $A$ to be closed in $A$, it suffices that $A_{0}$ be closed with respect to all linear systems containing no equations 3.1.

Proof. Let this condition be satisfied. Then $A_{0}$ is, in particular, closed with respect to all (single) congruences of the form $0 \cdot x \equiv a(\bmod r)$; which implies that $A_{0}$ is serving in $A$. Now let $L$ be a linear system, with all its constants $a_{i}$ in $A_{0}$, which has a solution in $A$ (and, hence, is not of type III). We have to show that $L$ can already be solved in $A_{0}$. If $L$ contains no equations 3.1 , this follows from our assumption. If $L$ is of type $I$, it follows from 3.8. Finally, if $L$ is of type II, we can replace it by a conjugate system $L^{\prime}$ with no equations in it (see 3.6). Since conjugate systems are solvable in exactly the same ordered groups, both $L$ and $L^{\prime}$ have a solution in $A$ and, hence (by our assumption), in $A_{0}$.

4. Linear systems in regularly ordered groups. Our aim in this section is to prove Theorem 3.7 of [3]. First, however, we shall consider a special case of it.

4.1. TheOREm. If $A_{0}$ and $A$ are regularly discrete groups having the same unit $\mathrm{i}$, and $A_{0} \subseteq A$, then $A_{0}$ is closed in $A$, with respect to all linear systems in one unknown $x$.

Proof. Let $L$ be such a system, with its constants $a_{i}$ in $A_{0}$, having a solution $x=c$ in $A$. We have to show that $L$ can already be solved in $A_{0}$. Without loss of generality, we may assume (by a similar argument as in 3.9) that $L$ contains no equations. Thus $L$ has the form
4.1.1.
$q_{i} x>a_{i}$,
$i=m_{1}+1, \cdots, m_{2}$,
4.1.2.
$q_{i} x \equiv a_{i}\left(\bmod r_{i}\right)$,
$i=m_{2}+1, m_{2}+2, \cdots, m_{3}$,
4.1.3.
$q_{i} x \not \equiv a_{i}\left(\bmod r_{i}\right)$,
$i=m_{3}+1, m_{3}+2, \cdots, m_{4}$.

By multiplying these formulae by suitable integers, we can make all $r_{i}$ equal, say, to $r$, and also make all $q_{i}$ in the inequalities 4.1.1 equal, say, to 
$q>0$, reversing, if necessary, the inequality signs. Thus 4.1 .1 is replaced by two sets of inequalities, of the form $q x>b_{i}$ and $q x<d_{j}\left(q>0 ; b_{i}, d_{j} \in A_{0}\right)$. Assuming that both sets are nonempty (otherwise only insignificant changes are required), we set $b=\max b_{i}$, and $d=\min d_{j}$. Then 4.1 .1 is equivalent to
4.1.4.
$b<q x<d$
$\left(q>0 ; b, d \in A_{0}\right)$.

Since $x=c$ is a solution of $L$, we have, in particular,

$$
\text { 4.1.5. } \quad b<q c<d \text {. }
$$

As $A_{0}$ is basic in $A$ (by 1.2$)$, there is an element $c^{\prime} \in A_{0}$ with $c^{\prime} \equiv c(\bmod r)$, where $r$ is the common value of the $r_{i}$. Clearly, $c^{\prime}$ satisfies 4.1.2 and 4.1.3 (for so does $c$ ). Fixing this $c^{\prime} \in A_{0}$, we now consider two possible cases:

(I) The interval $(b, d)$ is infinite $\left.{ }^{11}\right)$. Then, by 0.2 , it contains an element $a \in A_{0}$, of the form $a=q x_{0}$, with $x_{0} \equiv c^{\prime}(\bmod r), x_{0} \in A_{0}, b<q x_{0}<d$. As is readily seen, $x_{0}$ satisfies both 4.1 .4 and $4.1 .2-4.1 .3$. Thus $x=x_{0}$ is the required solution of $L$ in $A_{0}$.

(II) The interval $(b, d)$ is finite. Then all its elements have the form $b+k \cdot \dot{1}$ and, hence, belong to $A_{0}$ (for so does $b$ ). From 4.1.5 it then follows that $q c \in A_{0}$. Moreover, $q c$ is, clearly, divisible by $q$ in $A$. As $A_{0}$ is serving in $A$ (by 1.2), this implies that the quotient $(q c) / q=c$ is an element of $A_{0}$. Thus, in this case, $x=c \in A_{0}$ is already the required solution of $L$ in $A_{0 .}$ ( ${ }^{12}$ ) This completes the proof.

4.2. Lemma. Let $A_{0}$ and $A\left(A_{0} \subseteq A\right)$ be regularly discrete groups having the same unit $\mathrm{i}$, and let $c_{0} \in A$. Then $A_{0}$ is a closed subgroup of its $c_{0}$-extension in $A$ (call that extension $A_{1}$ ).

Proof. Let $L$ be a linear system in $n$ unknowns $x_{j}$, with its constants $a_{i}$ in $A_{0}$, and let $\left(c_{1}, \cdots, c_{n}\right)$ be its solution in $A_{1}$. We shall show, in three steps, that $L$ can already be solved in $A_{0}$, which will complete the proof.

(I) By the definition of a $c_{0}$-extension $\left(\$ 1\right.$, Part IV), the elements $c_{j}$ satisfy some equations of the form

$$
t_{j} c_{j}=a_{j} c_{0}+b_{j} \quad\left(b_{j} \in A_{0}\right), j=1,2, \cdots, n,
$$

with $t_{j}$ and $s_{j}$ integers $\left(t_{j}>0\right)$. As $\left(c_{1}, \cdots, c_{n}\right)$ is a solution, we may substitute the $c_{j}$ for the $x_{j}$ in $L$, thus obtaining a set of correct formulae. Using 4.2.1, we then eliminate $c_{1}, c_{2}, \cdots, c_{n}$ from these formulae $\left({ }^{13}\right)$. After simplifications, this process yields a set of (correct) formulae, of the form

$$
\text { 4.2.2. } \quad k_{i} c_{0}=a_{i}^{\prime}, \quad i=1,2, \cdots, m_{1} \text {, }
$$

(11) Note that $(b, d)$ is infinite in $A_{0}$ if and only if it is infinite in $A$.

(12) Recall that $x=c$ was supposed to be a solution of $L$.

(18) Footnote 8 applies to this elimination process as well, with $k$ replaced by some common multiple of the $t_{j}$, and other selfevident modifications. 
4.2 .3

4.2 .5 .

$$
k_{i} c_{0}>a_{i}^{\prime},
$$$$
k_{2} c_{0} \equiv a_{i}^{\prime}\left(\bmod r_{i}^{\prime}\right) \text {, }
$$$$
k_{2} c_{0} \not \equiv a_{i}^{\prime}\left(\bmod r_{i}^{\prime}\right) \text {, }
$$

$$
\begin{aligned}
& i=m_{1}+1, m_{1}+2, \cdots, m_{2}, \\
& i=m_{2}+1, m_{2}+2, \cdots, m_{3},
\end{aligned}
$$$$
i=m_{3}+1, m_{3}+1, \cdots, m_{4} \text {, }
$$

with $k_{i}, r_{i}^{\prime}$ integers $\left(r_{i}^{\prime}>0\right)$, and all $a_{i}^{\prime}$ in $A_{0}$.

(II) We now introduce an auxiliary linear system $L^{\prime}$ which arises from formulae 4.2.1 through 4.2.5 if the $c_{j}$ (including $c_{0}$ ) are replaced by $n+1$ unknowns, $x_{0}, x_{1}, \cdots, x_{n}$. In particular, equations 4.2.1 transform into
$4.2 .1^{\circ}$.
$t_{j} x_{j}=s_{j} x_{0}+b_{j}$
$\left(b_{j} \in A_{0}\right), j=1,2, \cdots, n$.

Clearly, $\left(c_{0}, c_{1}, \cdots, c_{n}\right)$ is a solution of $L^{\prime}$ in $A_{1}$. Moreover, if $\left(d_{0}, \cdots, d_{n}\right)$ is any solution of $L^{\prime}$ in $A$, then $\left(d_{1}, \cdots, d_{n}\right)$ is a solution of $L$; for the whole process described in step (I) is reversible (all groups being torsion-free). To prove the theorem, it then suffices to show that $L^{\prime}$ has a solution in $A_{0}$.

(III) For this purpose, we use $4.2 .1^{\circ}$ to eliminate the unknowns $x_{1}, \cdots, x_{n}$ from $L^{\prime}$, leaving only $x_{0}$ in it, and then replace equations $4.2 .1^{\circ}$ by a conjugate set of congruences, $s_{j} x_{0}+b_{j} \equiv 0\left(\bmod t_{j}\right), j=1,2, \cdots, n$, where the $s_{j}, b_{j}$ and $t_{j}$ are as in $4.2 .1^{\circ}$. This yields a linear system $L^{\prime \prime}$ in one unknown $x_{0}$ only; $L^{\prime \prime}$ consists of the now adjoined congruences and 4.2.2-4.2.5 (with $c_{0}$ replaced by $x_{0}$ ). An argument analogous to that of 3.6 shows that $L^{\prime}$ and $L^{\prime \prime}$ are conjugate systems. Moreover, $L^{\prime \prime}$ has a solution in $A_{1}$ (e.g., $\left.x_{0}=c_{0}\right)$. Therefore, by $4.1, L^{\prime \prime}$ (and, hence, also $L^{\prime}$ ) can be solved in $A_{0}$ as well (in fact, by 2.1.1, $A_{1}$ is regularly discrete and has the same unit as $A_{0}$; so all assumptions of 4.1 are satisfied). This completes the proof. We are now able to prove Theorem 3.7 of [3], even in a somewhat stronger version:

4.3. Theorem. Let $A_{0}$ and $A\left(A_{0} \subseteq A\right)$ be regularly discrete groups. Then $A_{0}$ is closed in $A$ if and only if the two groups have the same unit, $\dot{1}$.

Proof. (i) Necessity. If the units of $A_{0}$ and $A$ are different, then, clearly, $A_{0}$ has the larger unit. Hence, the system of inequalities $0<x<\mathrm{i}\left(\mathrm{i} \in A_{0}\right)$ can be solved in $A$, but not in $A_{0}$. Thus $A_{0}$ is not closed in $A$.

(ii) Sufficiency. Let $A_{0}$ and $A$ have the same unit, and let $L$ be a linear system, with its constants in $A_{0}$, and with a solution, $\left(c_{1}, c_{2}, \cdots, c_{n}\right)$, in $A$ (here $n$ is the number of the unknowns $x_{j}$ ). We have to show that $L$ can already be solved in $A_{0}$. For this purpose, we inductively define $n$ additional subgroups $A_{j}$, setting: $A_{j}$ equals the $c_{j}$-extension of $A_{j-1}$ in $A(j=1,2, \cdots, n)$. By 2.1.1 and 1.2, all $A_{j}$ are regularly discrete and serving subgroups of $A$, and have the same unit as $A$ and $A_{0}$. Moreover, $A_{n}$ contains all $c_{j}$; hence $L$ has in $A_{n}$ the same solution, $\left(c_{1}, \cdots, c_{n}\right) .\left({ }^{14}\right)$ By applying Lemma 4.2 recursively $n$ times, we infer that $L$ has a solution in $A_{0}$ as well, q.e.d.

The same method of proof (i.e., first proving the required theorems for

(14) Recall that, by $1.2, A_{n}$ is a serving subgroup of $A$. Therefore the congruences and incongruences contained in $L$ are satisfied, both in $A$ and $A_{n}$, by the same elements $c_{1}, c_{2}, \cdots, c_{n}$, supposed to constitute a solution of $L$. 
systems in one unknown, then for $c_{0}$-extensions, and, finally, generalizing them by introducing the subgroups $A_{j}$ as in 4.3) can also be used to establish the following two propositions (the details of the proof will be omitted) $\left({ }^{15}\right)$.

4.4. Every divisible subgroup $A_{0}$ of a divisible ordered group $A$ is closed in $A$, with respect to all systems of inequalities of the form 3.2.

4.5. If $A_{0}$ is a serving subgroup of an ordered group $A$ and has the same prime invariants as $A$, then $A_{0}$ is closed in $A$, with respect to all systems of congruences and (or) incongruences of the form 3.3 and 3.4 .

Our next objective is to prove a theorem analogous to 4.3 for regularly dense groups (this theorem is, likewise, a prerequisite of [3]). We need two lemmas.

4.6. Lemma. Let $\left(c_{1}, \cdots, c_{n}\right)$ be a solution of a system $L^{\prime}$ of inequalities of the form 3.2, in a densely ordered group $A$. Then $A$ contains a positive element $d$ such that the system $L^{\prime}$ is also satisfied by every other $n$-tuple of elements $\left(b_{1}, \cdots, b_{n}\right)$, with $c_{j}-d<b_{j}<c_{j}+d, b_{j} \in A, j=1,2, \cdots, n$.

For the proof, let $D>0$ be the least among the (positive) expressions $\sum_{j=1}^{n} q_{i j} c_{j}-a_{i}, i=m_{1}+1, m_{1}+2, \cdots, m_{2}$, with the $q_{i j}$ and $a_{i}$ as in 3.2 ; also let $q=\max \left|q_{i j}\right|$. By 2.3 , there exists a positive element $d \in A$, with $0<n q d$ $<D$. Then it is easily verified that the element $d$ has the required property.

4.7. Leмma. Every regularly dense subgroup $A_{0}$ of an ordered group $A$ is closed in $A$, with respect to all systems of inequalities of the form 3.2.

Proof. Let $M_{0}$ and $M\left(M_{0} \subseteq M\right)$ be the divisible extensions of $A_{0}$ and $A$, respectively (see $\$ 1$, Part V). Also let $L$ be a system of inequalities of the form 3.2, with all $a_{i}$ in $A_{0}$, which has a solution in $A$ and, a fortiori, in $M$. By 4.4, $L$ has also a solution in $M_{0}$. This solution has the form $x_{j}=c_{j} / k(j=1,2, \cdots, n)$, with $k$ a positive integer and $c_{j} \in A_{0}$ (in fact, by the definition of a divisible extension, all elements of $M_{0}$ are such fractions; the number $k$ may be assumed to be the common denominator of the fractions involved). Substitution of the $c_{j} / k$ for the unknowns $x_{j}$ in 3.2 yields

4.7.1. $\quad \sum_{j=1}^{n} q_{i j} c_{j}>k a_{i}, \quad i=m_{1}+1, m_{1}+2, \cdots, m_{2} ; c_{j} \in A_{0}$.

Thus $\left(c_{1}, c_{2}, \cdots, c_{n}\right)$ is a solution, in $A_{0}$, of the auxiliary system

4.7.2.

$$
\sum_{j=1}^{n} q_{i j} x_{j}>k a_{i}, \quad i=m_{1}+1, m_{1}+2, \cdots, m_{2} .
$$

As $A_{0}$ is densely ordered, we can find a positive element $d \in A_{0}$ with properties specified in 4.6 (taking 4.7 .2 for $L^{\prime}$ ). Moreover, since the intervals $\left(c_{j}-d, c_{j}+d\right)$ are infinite, each of them contains (by the $k$-regularity of $A_{0}$ )

(15) Proposition 4.4 is actually a special case of A. Robinson's Theorem 3.1.5 proved in [2], and can also be proved in the same way as the latter. 
a $k$-divisible element, call it $k b_{j}\left(b_{j} \in A_{0}\right)$. Then, by $4.6,\left(k b_{1}, \cdots, k b_{n}\right)$ is another solution of 4.7.2 in $A_{0}$. Substituting the elements $k b_{j}$ for the unknowns $x_{j}$ in 4.7.2 and reducing by $k$, we see that $\left(b_{1}, \cdots, b_{n}\right)$ is a solution in $A_{0}$ of the original system $L$. This completes the proof.

We are now able to establish the required additional result.

4.8. Theorem. For a regularly dense subgroup $A_{0}$ of an ordered group $A$ to be closed in $A$, it suffices that $A_{0}$ be serving in $A$ and have the same prime invariants as $A$, i.e., $[p] A_{0}=[p] A$, for every prime $p .\left({ }^{16}\right)$

Proof. Let these conditions hold, and let $L$ be a linear system of the form $3.2-3.4$, with no equations in it $\left({ }^{17}\right)$, and with all its constants $a_{i}$ in $A_{0}$. Given that $L$ has a solution in $A$, we have to show that it can already be solved in $A_{0}$. By $4.7, A_{0}$ contains, in any case, a solution, $\left(b_{1}, \cdots, b_{n}\right)$, of the partial system 3.2, consisting of inequalities only. Similarly, by 4.5 , there is a solution, $\left(c_{1}, \cdots, c_{n}\right)\left(c_{j} \in A_{0}\right)$, of the partial system $3.3-3.4$, consisting of the congruences and incongruences contained in $L$. Fixing these two solutions, we now use 4.6 to select a positive element $d \in A_{0}$ such that the inequalities 3.2 of $L$ are also satisfied by every other $n$-tuple $\left(b_{1}^{\prime}, \cdots, b_{n}^{\prime}\right)$ with

$$
\text { 4.8.1. } \quad b_{j}^{\prime} \in\left(b_{j}-d, b_{j}+d\right), \quad b_{j}^{\prime} \in A_{0}, \quad j=1,2, \cdots, n \text {. }
$$

Now let $r>0$ be a common multiple of the moduli $r_{i}$ in 3.3-3.4. As $A_{0}$ is regularly dense, each of the infinite intervals $\left(b_{j}-d, b_{j}+d\right)$ contains an element $b_{j}^{\prime}$ with $b_{j}^{\prime} \equiv c_{j}(\bmod r)$ in $A_{0}, b_{j}^{\prime} \in A_{0}$ (see 0.2 , with $\left.k=1\right)$. Then it easily follows that the $b_{j}^{\prime}(j=1,2, \cdots, n)$ satisfy the congruences and incongruences of $L$ (for so do the $c_{j}$ ). As the $b_{j}^{\prime}$ also satisfy the conditions 4.8.1, they constitute a solution of the inequalities 3.2 as well. Thus $\left(b_{1}^{\prime}, \cdots, b_{2}^{\prime}\right)$ is a solution, in $A_{0}$, of the whole system $L$. This completes the proof.

5. Countable groups with the same prime invariants. $(D / n)$-extensions. In order to prove the last of the required theorems (3.8 of [3]), we need some additional auxiliary concepts and propositions.

I. Let $A$ and $B$ be two countable disjoint torsion-free (possibly ordered) but not completely divisible groups such that $[p] A=[p] B$ for every prime p. $\left({ }^{18}\right)$ Let

5.1 .

$$
p_{1}<p_{2}<p_{3}<\cdots<p_{k}<\cdots
$$

be the (finite or infinite) sequence of all primes which do not divide $A$ and $B$. Using 1.7, select, for each $k$, a nonvoid maximal $p_{k}$-independent subset $S_{k} \subseteq A$, and also such a subset $T_{k} \subseteq B$. Due to countability, each $S_{k}$ and $T_{k}$ can be written as a (finite or infinite) sequence, say

(16) These conditions can also easily be proved to be necessary.

(17) We lose no generality by making this assumption (see 3.9).

(18) Note that, due to this assumption, $A$ and $B$ are divisible by exactly the same primes (for, $p$ divides a group if and only if $[p]=1$ in that group). 


$$
S_{k}=\left\{a_{k i}\right\}, \quad T_{k}=\left\{b_{k i}\right\}, \quad a_{k i} \in A, b_{k i} \in B .
$$

Since $\left[p_{k}\right] A=\left[p_{k}\right] B$, Corollary 1.8 implies that each $S_{k}$ contains exactly as many elements as does the corresponding $T_{k}$, namely $\log _{p_{k}}\left[p_{k}\right] A$ $=\log _{p_{k}}\left[p_{k}\right] B$. The one-to-one correspondence between $S_{k}$ and $T_{k}$ is, obviously, effected by the mapping $a_{k i} \rightarrow b_{k i}$ (see 5.2). Therefore, by embedding $A$ and $B$ in their direct sum $M_{0}$, we can define, for each $k$, a set $D_{k}$ consisting of the differences of the form $a_{k i}-b_{k i}$, i.e.,

$$
D_{k}=\left\{a_{k 1}-b_{k 1}, a_{k 2}-b_{k 2}, \cdots, a_{k i}-b_{k i}, \cdots\right\} .
$$

The sets $S_{k}, T_{k}, D_{k}$ and the sequences 5.2 and 5.3 will henceforth be considered as fixed. The subgroups generated by $S_{k}, T_{k}, D_{k}$ will be denoted by $\bar{S}_{k}, \bar{T}_{k}, \bar{D}_{k}$, respectively. With these conventions, we have: tively.

5.4. Theorem. The subgroups $\bar{S}_{k}$ and $\bar{T}_{k}$ are $p_{k}$-basic in $A$ and $B$, respec-

We carry out the proof for $\bar{S}_{k}$. Suppose that $\bar{S}_{k}$ is not $p_{k}$-basic in $A$. Then there is an element $y \in A$ which is $p_{k}$-incongruent with any element of $\bar{S}_{k}$, so that, certainly, $y \notin S_{k}$. The maximality of $S_{k}$ then implies that $\left(S_{k} \cup\{y\}\right)$ is not $p_{k}$-independent in $A$, so that, for some integers $q_{i}$, and some distinct elements $x_{i} \in S_{k}$, we have

$$
q_{0} y+\sum_{i=1}^{m} q_{i} x_{i} \equiv 0\left(\bmod p_{k}\right)
$$

$q_{0} \not \equiv 0\left(\bmod p_{k}\right)$

As $q_{0}$ is prime to $p_{k}$, there are integers $s, t$ with $y=s q_{0} y+p_{k} t y$, i.e., $y \equiv s q_{0} y\left(\bmod p_{k}\right)$ whence, by 5.4.1,y $\equiv-s \sum_{i=1}^{m} q_{i} x_{i}\left(\bmod p_{k}\right), x_{i} \in S_{k}$, which is impossible since $y$ is $p_{k}$-incongruent with every element of $\bar{S}_{k}$.

5.5. Theorem. Each $D_{k}$ is $p_{k}$-independent in the direct sum $M_{0}$ of $A$ and $B$.

Proof. By $1.10(\mathrm{c}, \mathrm{d})$, the relation $\sum_{i=1}^{m} q_{i}\left(a_{k i}-b_{k i}\right) \equiv 0\left(\bmod p_{k}\right)$ in $M_{0}$ implies $\sum_{i=1}^{m} q_{i} a_{i} \equiv 0\left(\bmod p_{k}\right)$ in $A$ whence (due to the $p_{k}$-independence of $S_{k}$ in $A) q_{i} \equiv 0\left(\bmod p_{k}\right)$, as required.

5.6. TheOREM. For each $p_{k}$ and any elements $d \in D_{k}, a \in A$ (or $a \in B$ ), the relation $a \equiv d\left(\bmod p_{k}\right)$ in $M_{0}$ implies $a \equiv 0\left(\bmod p_{k}\right)$ in $A($ in $B$, respectively).

Proof. Let $a \equiv d\left(\bmod p_{k}\right)$ in $M_{0}$ where $a \in A$ and $d=\sum_{i=1}^{m} q_{i}\left(a_{k i}-b_{k i}\right)$. By 5.4, $a$ is $p_{k}$-congruent with some element of $\bar{S}_{k}$, say with $\sum_{i=1}^{m} r_{i} a_{k i}$, where $m$ and the $a_{k i}$ may be assumed to be the same as in the expression for $d$ (we need only use some zero-coefficients). We then have $a \equiv \sum_{i=1}^{m} r_{i} a_{k i}$ $\equiv \sum_{i=1}^{m} q_{i}\left(a_{k i}-b_{k i}\right)\left(\bmod p_{k}\right)$ in $M_{0}$. These relations imply, by $1.10(\mathrm{c}, \mathrm{d})$ that

$$
a \equiv \sum_{i=1}^{m} r_{i} a_{k i} \equiv \sum_{i=1}^{m} q_{i} a_{k i}\left(\bmod p_{k}\right) \text { in } A
$$


and

5.6 .3

$$
\sum_{i=1}^{m} q_{i} b_{k i} \equiv 0\left(\bmod p_{k}\right) \text { in } M_{0} \text { and } B \text {. }
$$

As $T_{k}$ is $p_{k}$-independent in $B, 5.6 .3$ implies $q_{i} \equiv 0\left(\bmod p_{k}\right)$ for all $q_{i}$. This, combined with 5.6.2, yields $a \equiv 0\left(\bmod p_{k}\right)$, as required.

II. Now let $M^{\prime}$ be a divisible group, $M$ its subgroup, $D$ a nonempty subset of $M$, and $\bar{D}$ the subgroup generated by $D$. For every integer $n>0$, we define the $(D / n)$-extension of $M$ in $M^{\prime}$ to be the set $M_{n}$ of all elements $u \in M^{\prime}$ with

$5.6^{*}$.

$u=x+\left(d / n^{k}\right)$

$(x \in M, d \in \bar{D}, k=0,1,2, \cdots)$,

where $d / n^{k}$ is any one of the quotients of $d$ by $n^{k}$ in $M^{\prime} .{ }^{(19)}$

5.7. Theorem. Let $M_{n}$ be the $(D / n)$-extension of $M$ in $M^{\prime}$, as above. Then: (a) $M_{n}$ is a subgroup of $M^{\prime}$ which contains $M$; (b) In $M_{n}$, all elements of $D$ are divisible by any powers of $n$; (c) $M$ is $q$-serving in $M_{n}$ whenever the integer $q$ is prime to $n$; (d) $M$ is basic in $M_{n}$.

Proof. Assertions (a) and (b) immediately follow from 5.6*. To prove (c), suppose that $y \equiv z(\bmod q)$ in $M_{n}$ for some $y, z \in M$, with $q$ prime to $n$. Then $y-z=q u\left(u \in M_{n}\right)$, whence, by $5.6^{*}, n^{k}(y-z)=q n^{k} u=q\left(n^{k} x+d\right),(x, d \in M)$, so that $q$ divides $n^{k}(y-z)$ in $M$. As $n^{k}$ is prime to $q, q$ must divide $y-z$ in $M$. Thus the congruence $y \equiv z(\bmod q)$ holds in $M$ whenever it holds in $M_{n}$, q.e.d. For the proof of (d), it suffices to show that $M$ is $p$-basic in $M_{n}$ for every prime $p$ (see 1.3). If $p$ divides $n, 5.6^{*}$, combined with $5.7(\mathrm{~b})$, easily implies that every $u \in M_{n}$ is $p$-congruent (in $M_{n}$ ) to some $x \in M$, as required. If, however, $p$ does not divide $n$, the same fact is obtained by combining $5.6^{*}$ with the equation $p s+n^{k} t=1$ ( $s, k$ are integers).

5.8. Theorem. With the same notation, if $M^{\prime}$ is torsion-free, and $D$ is $n$ independent in $M$, then, for any $y \in M$ to be divisible by $n$ in $M_{n}$, it is necessary and sufficient that $y$ be $n$-congruent (in $M$ ) with some element of $\bar{D}$.

Proof. Sufficiency follows from 5.7(b). To prove necessity, let $n$ divide the element $y \in M$ in $M_{n}$; i.e., let $y=n u\left(u \in M_{n}\right)$. Then $5.6^{*}$ yields

$$
\text { 5.8.1. } \quad y-n x=d / n^{k-1} \quad(x, y \in M, d \in \bar{D}) \text {; }
$$

that is, $d$ is divisible by $n^{k-1}$ in $M$. As $d \in \bar{D}$, we have

$$
d=\sum_{i=1}^{m} q_{i} d_{i}
$$

for some integers $q_{i}$, so that the divisibility of $d$ by $n^{k-1}$ implies

(19) Obviously, the expression $d / n^{k}$ may be many-valued if $M^{\prime}$ is not torsion-free. This is why several quotients of $d$ by $n^{k}$ may be involved. 
5.8 .3 .

$$
\sum_{i=1}^{m} q_{i} d_{i} \equiv 0\left(\bmod n^{k-1}\right) \text { in } M
$$

But $D$ is, by assumption, $n$-independent in $M$. As $M$ is torsion-free, it follows, by 1.6 , that $D$ is also $n^{k-1}$-independent in $M$. Therefore relation 5.8.3 implies that all $q_{i}$ are divisible by $n^{k-1}$. Hence (see 5.8.2) $n^{k-1}$ divides $d$ in $\bar{D}$, i.e., $d=n^{k-1} d^{\prime}$, for some $d^{\prime} \in \bar{D}$. This, combined with 5.8 .1 , yields $y \equiv d^{\prime}(\bmod n)$ in $M$, as asserted.

After this preparatory work, we are now able to prove the last of the required results, namely, Theorem 3.8 of [3]. We shall, however, split it into two theorems (6.1 and 6.3) the first of which is more general (since it also applies to unordered groups), whereas the second is the one postulated in [3], with regard to regularly dense groups.

\section{Two embedding theorems.}

6.1. Theorem. Let $A$ and $B$ be two countable disjoint torsion-free (ordered) abelian groups, with $[p] A=[p] B$, for every prime $p$. Then there exists a torsionfree (ordered, respectively) group $M$ such that (a) $A$ and $B$ are serving and basic subgroups in $M$; (b) $M$ has the same prime invariants as $A$ and $B$, i.e., [p]M $=[p] A=[p] B$, for every prime $p \cdot\left({ }^{20}\right)$

Proof. Assertion (b) follows from (a), by 1.4 ; so it suffices to prove (a). Furthermore, it suffices to consider the case where $A$ and $B$ are not divisible (otherwise, their direct sum can serve as the required group $M$ ).

Let then again 5.1 be the sequence of the primes $p_{k}$ which do not divide $A$ and $B$, and let the sets $S_{k}, T_{k}, D_{k}$ and the groups $\bar{S}_{k}, \bar{T}_{k}, \bar{D}_{k}$ be defined as in $\S 5$. Further, let $M^{\prime}$ be the divisible extension of the direct sum $M_{0}$ of $A$ and $B$. We start with constructing an ascending sequence of groups

6.1.1.

$$
M_{0} \subseteq M_{1} \subseteq \cdots \subseteq M_{k} \subseteq \cdots,
$$

where $M_{k}$ is defined inductively by

$$
\text { 6.1.2. } M_{k} \text { is the }\left(D_{k} / p_{k}\right) \text {-extension of } M_{k-1} \text { in } M^{\prime}, \quad k=1,2,3, \cdots
$$

The inductive process 6.1.2 is infinite or finite according as the sequence 5.1 is infinite or finite. As is readily seen, each $M_{k}$ contains $A$ and $B$, and is a subgroup of $M^{\prime}$. As $M^{\prime}$ is torsion-free (ordered), so are all $M_{k}$. The divisible extension of each $M_{k}$ coincides with $M^{\prime}$. Finite induction easily yields the following facts.

6.1.3. $M_{0}$ is a basic subgroup in each $M_{k}$. (For the proof, apply $5.7(\mathrm{~d})$. )

6.1.4. $M_{k}$ is p-serving in $M_{k+n}(n=1,2, \cdots)$ for every prime $p$ other than $p_{k+i}(i=1,2, \cdots, n)$. (Apply 5.7(c).)

6.1.5. If $A$ and $B$ are divisible by some prime $p$, so is each $M_{k}$. (Apply 5.6*.) in $M$.

(20) The zero elements of $A$ and $B$ must, of course, be identified when they are embedded 
6.1.6. Each $D_{k}$ is $p_{k}$-independent in $M_{n}$, for $n=0,1, \cdots, k-1$. (Use 5.5 and $5.7(\mathrm{c})$.

6.1.7. $A$ and $B$ are serving in each $M_{k}$. (Apply 1.3, 1.10(d), 5.8 and 5.6.) Furthermore, we have:

6.1.8. If $p$ is one of the primes $p_{k}(k=1,2, \cdots, n)$, then: (a) For every element $a \in A(a \in B)$, there is an element $b \in B(b \in A)$ with $a \equiv b(\bmod p)$ in $M_{n}$. (b) $A$ and $B$ are $p$-basic in $M_{n}$.

In fact, if $p=p_{n}$, every element $a \in A$ is, by 5.4 , $p$-congruent to some element of $\bar{S}_{n}$, say

$6.1 .8^{\prime}$

$$
a \equiv \sum_{i=1}^{m} q_{i} a_{n i}(\bmod p) \text { in } A \text { and in } M_{n} .
$$

Now take, for each $a_{n i}$, the corresponding $b_{n i}$ (see 5.2), and let

$$
b=\sum_{i=1}^{m} q_{i} b_{n i}, \quad d=\sum_{i=1}^{m} q_{i}\left(a_{n i}-b_{n i}\right), \quad b \in B, d \in \bar{D}_{n} .
$$

This, combined with $6.1 .8^{\prime}$ yields $d \equiv a-b(\bmod p)$ in $M_{n}$, whence, by 5.7(b),$a-b \equiv 0 \equiv d(\bmod p)$ in $M_{n}$, as asserted. If, however, $p=p_{k}(1 \leqq k<n)$ then, by what has been proved, for every $a \in A$, there is a $b \in B$ with $a \equiv b(\bmod p)$ in $M_{k}$. As $M_{k} \subseteq M_{n}$, this congruence holds in $M_{n}$ as well. This establishes (a). Now take any element $u \in M_{n}$. By 6.1.3 and 1.10(b), there are elements $x \in A$ and $y \in B$ such that

$$
\text { 6.1.8". } \quad u \equiv x+y(\bmod p) \text { in } M_{n}, \quad(x+y) \in M_{0} \text {. }
$$

On the other hand, by $(\mathrm{a})$, we have $y \equiv x^{\prime}(\bmod p)$ in $M_{n}$, for some $x^{\prime} \in A$. This, combined with $6.1 .8^{\prime \prime}$, yields $u \equiv x+x^{\prime}(\bmod p)$, where $\left(x+x^{\prime}\right) \in A$. Thus every element $u \in M_{n}$ is $p$-congruent with some element of $A$, i.e., $A$ is $p$-basic in $M_{n}$. Similarly for $B$.

Now let $M$ be the union of all $M_{k}$. Then the group $M$ is torsion-free (ordered, respectively), for $M \subseteq M^{\prime}$. From 6.1.7 it easily follows that $A$ and $B$ are serving subgroups of $M$. By 1.3 , it remains to show that $A$ and $B$ are $p$-basic in $M$, for all primes values of $p$. Let then $p$ be any prime, and $u \in M$. We consider two cases.

(I) $p$ is none of the primes $p_{k}$. Then $A$ and $B$ are divisible by $p$; so are also all $M_{k}$ (see 6.1.5). Hence, $M$ too is divisible by $p$, so that all elements of $M$ are $p$-congruent. It follows (trivially) that $A$ and $B$ are $p$-basic in $M$.

(II) $p$ is one of the primes $p_{k}$, say $p=p_{k_{0}}$. By assumption, $u \in M$, i.e., $u$ belongs to some $M_{k}$, say $u \in M_{n}$. If $k_{0} \leqq n$, then 6.1 .8 implies that $A$ and $B$ are $p$-basic in $M_{n}$, so that $u$ is $p$-congruent with some $x \in A$ and some $y \in B$. If, however, $n<k_{0}$, then $M_{n} \subseteq M_{k_{0}}$, and the same conclusion follows from 6.1.8. Thus, in all possible cases, $u$ is p-congruent with some elements of $A$ and $B$. This shows that $A$ and $B$ are basic in $M$, so that the group $M$ has all the required properties. This completes the proof. 
6.2. Note. It follows from this proof that the group $M$ has the following additional properties.

(i) Every element $u \in M$ satisfies some equation of the form $n u=a+b$ where $a \in A, b \in B$, and $n$ is a positive integer.

(ii) If $A$ and $B$ are ordered, so is $M$. Moreover, the elements of $A$ and $B$ follow each other in $M$ in the same order as they do in the direct sum $M_{0}$ of $A$ and $B$ (ordered lexicographically).

Both properties follow from the fact that $M$ is a subgroup of $M^{\prime}$ in which they hold. In the sequel, we shall assume that $M_{0}$ is ordered in such a way that the positive elements of $A$ are greater than those of $B$ (this amounts to selecting one of the two possible lexicographic orderings in $M_{0}$ ).

6.3. Theorem. Let $A$ and $B$ be two disjoint countable regularly dense groups having the same prime invariants. Then they can always be embedded in a regularly dense group $M$ in such a way that $M$ has the same prime invariants as $A$ and $B$, and contains $A$ and $B$ as serving and basic subgroups.

Proof. Construct the group $M$ exactly as in Theorem 6.1. Then $M$ has the same prime invariants as $A$ and $B$, and contains $A$ and $B$ as serving and basic subgroups. It remains to show that $M$ is regularly dense. Let then $q$ be an integer $>0$, and $u_{1}, u_{2}\left(u_{1}<u_{2}\right)$ two elements of $M$. By 6.2(i), we have $n_{1} u_{1}$ $=a_{1}+b_{1}$ and $n_{2} u_{2}=a_{2}+b_{2}\left(a_{1}, a_{2} \in A ; b_{1}, b_{2} \in B\right)$; obviously, we may set $n_{1}$ $=n_{2}=n$. Thus

6.3.1. $n u_{1}=a_{1}+b_{1}, \quad n u_{2}=a_{2}+b_{2} ; a_{1}, a_{2} \in A ; \quad b_{1}, b_{2} \in B ; n>0$.

As $u_{1}<u_{2}$, we have $a_{1}-a_{2}<b_{2}-b_{1}$ so that $a_{1}-a_{2}$ cannot be positive (this would contradict our convention as to the ordering of $M$ ). Consider two cases:

(I) $a_{1}-a_{2}<0$. In this case, due to the regular density of $A$, there is an element $y \in A$ with $a_{1}<n q y<a_{2}$ (see 2.1(a)). Again, our convention as to the ordering of $M$ easily implies that $a_{1}+b_{1}<n q y<a_{2}+b_{2}$, or, by $6.3 .1, u_{1}<q y<u_{2}$ $(y \in M)$, which is the required condition, by $2.1(\mathrm{a})$.

(II) $a_{1}=a_{2}=a$. In this case, using 6.3 .1 , we obtain

$$
n u_{1}=a+b_{1}, \quad n u_{2}=a+b_{2}, \quad b_{1}<b_{2} .
$$

As $B$ is basic in $M, a$ is $(q n)$-congruent with some $b \in B$, so that $a=b+q n x$ $(x \in M)$ whence, by 6.3.2,

$$
\text { 6.3.3. } n u_{1}=\left(b+b_{1}\right)+q n x, \quad n u_{2}=\left(b+b_{2}\right)+q n x, \quad b_{1}<b_{2} \text {. }
$$

Here $b+b_{1}<b+b_{2}$ and $b, b_{1}, b_{2} \in B$. Since $B$ is regularly dense, there is an element $x^{\prime} \in B$ with $b+b_{1}<q n x^{\prime}<b+b_{2}$. This, combined with 6.3.3, yields $u_{1}<q\left(x+x^{\prime}\right)<u_{2},\left(x+x^{\prime}\right) \in M$, and the regular density of $M$ is again established, by $2.1(\mathrm{a})$. Thus the theorem is proved.

Theorem 6.3 is a stronger version of the last of the required propositions (3.8 of [3]). Thus our objective of supplying the proofs of the theorems announced in [3] has been achieved. 


\section{REFERENCES}

1. H. Prüfer, Untersuchungen über die Zerlegbarkeit der abzählbaren primären abelschen Gruppen, Math. Z. vol. 17 (1923) pp. 35-61.

2. A. Robinson, Complete theories, Amsterdam, North-Holland Publishing Co., 1956.

3. A. Robinson and E. Zakon, Elementary properties of ordered abelian groups, Trans. Amer. Math. Soc. vol. 96 (1960) pp. 222-236.

4. W. Szmielew, Elementary properties of abelian groups, Fund. Math. vol. 41 (1955) pp. 203-271.

Essex College, Assumption University of Windsor, Windsor, Ontario, Canada 